\title{
PADRÃO REPRODUTIVO DE ELACHISTOCLEIS BICOLOR (ANURA, MICROHYLIDAE) NA SERRA DA BODOQUENA, MATO GROSSO DO SUL, BRASIL
}

\author{
Domingos de Jesus Rodrigues ${ }^{1}$ \\ Frederico Santos Lopes ${ }^{2}$ \\ Masao Uetanabaro ${ }^{2}$
}

\begin{abstract}
REPRODUCTIVE PATTERN OF ELACHISTOCLEIS BICOLOR (ANURA, MICROHYLIDAE) AT SERRA DA BODOQUENA, MATO GROSSO DO SUL, BRAZIL. The reproductive pattern of Elachistocleis bicolor (Guérin Méneville, 1838) was studied at Serra da Bodoquena, from October 2000 to September 2001. Reproduction occurred in the wet season (October to April) and was correlated to high continuous pluviometric precipitation. The species presents sexual size dimorphism, with females larger than males. The number of mature eggs per ovary was $620 \pm 251(\mathrm{n}=39)$ and mature eggs measured $1.15 \pm 0.30 \mathrm{~mm}(\mathrm{n}=40)$. Elachistocleis bicolor presented significant relations between snout-vent length and number of mature eggs $\left(n=39 ; r^{2}=0.25 ; p=0.001\right)$, individual weight and number of mature eggs $\left(n=41, r^{2}=0.30 ; p=0.002\right)$, snout-vent length and ovarian weight $\left(n=35 ; r^{2}=0.47 ; p<0.01\right)$, and individual weight and ovarian weight $\left(n=36 ; r^{2}=0.55 ; p<0.01\right)$. Weight and volume are better to study size-fecundity relationships than snout-vent length. The females invested $22.7 \pm 6.3 \%(\mathrm{n}=35)$ of their weights in reproduction and the variance associated to this variable was high, related to the reproductive mode of the species.
\end{abstract} Bodoquena.

KEYWORDS. Amphibia, Microhylidae, Elachistocleis, reproductive pattern, Serra da

\section{INTRODUÇÃO}

Para estudos de padrões reprodutivos de anuros, a facilidade de captura e, em especial, a fecundação externa permitem a quantificação bastante precisa do sucesso reprodutivo dos indivíduos (HowARD, 1988), facilitada ainda pelo desenvolvimento de técnicas para a marcação e identificação de indivíduos no campo (EMLEN, 1968). Na região Neotropical com mais de $50 \%$ das espécies de anuros conhecidas, ocorre a maior diversidade de modos reprodutivos (Duellman, 1999), que aparentemente está relacionada com a variedade de hábitats utilizados (Pough et al., 1998). Trabalhos sobre reprodução de anuros no Brasil (HödL, 1990; PrAdEIRO \& RoBInSON, 1990; Prado et al.,

1. Instituto Nacional de Pesquisas da Amazônia (INPA), Coordenação de Pesquisas em Ecologia, Campus V-8, Av. André Araújo, 2936,69011-970, Manaus, AM, Brasil. (poxo@inpa.gov.br)

2. Universidade Federal de Mato Grosso do Sul (UFMS), Caixa Postal, 549, 79070-900, Campo Grande, MS, Brasil. (fslopes@nin.ufms.br; masao@nin.ufms.br) 
2000) foram realizados, na maioria, na região Sudeste (BASTOS \& HADDAD, 1996; BERTOLUCI, 1998; Freitas et al., 2001) e na Floresta Amazônica (JunCÁ, 1998; Lima \& MAgnusson, 2000). O conhecimento sobre a herpetofauna do Cerrado é recente (Colli et al., 2002) e, apesar da intensa modificação ambiental provocada pela ação antrópica (BEEBEE, 1996), a biologia dos anuros permanece pouco conhecida (POMBAL \& BASTOS, 1996). Na Serra da Bodoquena, STRÜSSMANN et al. (2000) realizaram um levantamento das espécies de anuros, feito durante um programa de avaliação rápida da região pantaneira e Cerrado. São raros os estudos sobre esforço reprodutivo de espécies tropicais americanas (LAMPo \& MEDIALDEA, 1996; PRADO et al., 2000) e sua avaliação permite medir o custo da reprodução (STEARns, 1992). A maneira de mensurar tal esforço consiste em estabelecer proporções como o peso de gônadas em relação ao peso do corpo ou o volume da desova em relação ao volume do corpo (GADGIL \& BOSSERT, 1970; CRUMP, 1974; STEARNS, 1992). Em geral, o volume da desova está correlacionado com o tamanho da fêmea ou, mais diretamente, com o espaço disponível na sua cavidade abdominal (KAPLAN \& SaLthe, 1979).

Elachistocleis bicolor (Guérin Méneville, 1838) é um microhilídeo com comprimento rostro-cloacal entre $22-40 \mathrm{~mm}$, corpo ovóide, cabeça pequena e triangular e dorso castanho-escuro a castanho-amarelado com uma listra branca a amarelo-clara que se estende do nariz até a região do uróstilo. O ventre é amarelo imaculado e região gular preta nos machos e amarela nas fêmeas. Na porção posterior da coxa há uma linha estreita castanho-avermelhada (DE LA Riva et al., 1996; 2000; STRÜSSMANN et al., 2000; Frost, 2002).

O objetivo foi estudar a reprodução de E. bicolor em lagoas temporárias e áreas inundadas na Serra da Bodoquena, envolvendo período e padrão reprodutivo, tamanho da desova e dos óvulos maduros, investimento reprodutivo de fêmeas, ocorrência de dimorfismo sexual quanto ao comprimento rostro-cloacal (CRC) e massa corporal, e verificar se existe relação entre tamanho (CRC e peso do indivíduo) e fecundidade nas fêmeas grávidas.

\section{MATERIAL E MÉTODOS}

O trabalho foi realizado na Serra da Bodoquena, Fazenda Canaã $\left(20^{\circ} 40^{\prime} 30,4\right.$ '’; 56 $45^{\prime}$ '20,2”W) e Assentamento Canaã (20 41'32,3”S; 56 44'34,3”W), município de Bodoquena, Mato Grosso do Sul, Brasil. A temperatura média anual está entre 20 e $22^{\circ} \mathrm{C}$, e a precipitação média anual entre 1300 e $1700 \mathrm{~mm}$ (ALVARENGa et al., 1982). A maior incidência de chuvas ocorre nos meses de outubro a abril, com marcantes períodos de seca entre maio e setembro (Paulo César Boggiani, com. pess.).

O planalto da Bodoquena constitui feição de relevo localizada na porção centro-sul do Estado, na borda do Pantanal do Nabileque (Boggiani \& Clemente, 1999). A vegetação do planalto varia conforme o relevo e tipo de solo, onde na floresta semi-decídua pode-se encontrar espécies de angico (Anadenanthera sp.), capitão-do-campo (Terminalia argentea (Camb.) Mart.), gonçalo-alves (Astronium fraxinifolium Schott ex Spreng.), caraguatá (Bromelia balansae Mez), pau-terra-folhalarga (Qualea grandiflora Mart.), pau-terra-folha-miúda (Qualea parviflora Mart.), lixeira (Curatella americana L.), Bromeliaceae e, no campo, algumas espécies de Gramineae e Ciperaceae (FuRTADo et al., 1982). A lagoa estudada tinha aproximadamente $100 \mathrm{~m}^{2}$, profundidade de $20 \mathrm{a} 40 \mathrm{~cm}$ e vegetação formada por gramíneas e arbustos de Asteraceae. A área do campo inundado tinha aproximadamente $500 \mathrm{~m}^{2}$, profundidade de 15 a $80 \mathrm{~cm}$ e vegetação composta por gramíneas onde há o acumulo de água devido ao tipo de solo e posição da depressão (oeste da base do morro). A distância entre os dois pontos de coleta foi de aproximadamente $4 \mathrm{~km}$.

As coletas foram realizadas mensalmente em dois corpos d'água temporários, com permanência no campo, em média, de quatro dias, entre outubro de 2000 a setembro de 2001. Para cada espécime foram obtidos dados sobre o período de reprodução, local da desova e desenvolvimento dos girinos. $\mathrm{O}$ período reprodutivo foi determinado com base na vocalização dos machos, presença de casais em 
amplexo ou de girinos. A caracterização dos ambientes utilizados para a desova e desenvolvimento dos girinos foi feita anotando-se informações sobre a vegetação.

Os indivíduos coletados foram transportados em sacolas plásticas e congelados em refrigerador. O comprimento rostro-cloacal (CRC) dos indivíduos adultos foi medido com paquímetro de precisão $0,1 \mathrm{~mm}$. Utilizou-se uma balança digital de precisão $0,001 \mathrm{~g}$, para a pesagem dos indivíduos adultos (machos e fêmeas), bem como das gônadas maduras das fêmeas, que foram retiradas através de uma incisão ventro-lateral e pesadas individualmente. Os óvulos foram considerados maduros quando havia distinção entre o pólo animal e o vegetativo (CRUMP, 1974) e, neste caso, foram maiores que 0,6 $\mathrm{mm}$. O diâmetro dos óvulos foi medido com ocular micrométrica de precisão $0,1 \mathrm{~mm}$. O material foi identificado com base em De LA Riva et al. (1996; 2000) e Frost (2002) e depositado na Coleção Zoológica da Universidade Federal de Mato Grosso do Sul (UFMS 533-535).

Para verificar a influência do tamanho e massa corporal das fêmeas sobre a fecundidade, foram realizadas análises de regressão linear (ZAR, 1984) entre as seguintes variáveis: comprimento rostrocloacal (CRC) das fêmeas vs. número de óvulos maduros (NOM); peso dos indivíduos (PI) vs. NOM; CRC vs. peso dos ovários (PO); PI vs. PO. Para verificar a porcentagem de variação explicada pelo CRC e PI, utilizou-se o valor do $\mathrm{r}^{2}$ da análise de regressão. Quanto à existência de dimorfismo sexual em tamanho, aplicou-se o teste $\mathrm{t}$ de Student para a comparação dos comprimentos médios de machos e fêmeas adultos. O esforço reprodutivo das fêmeas foi medido como a porcentagem da massa dos ovários maduros em relação à massa bruta das fêmeas.

\section{RESULTADOS}

O período reprodutivo de E. bicolor iniciou em novembro estendendo-se até março e está relacionado com a ocorrência de altas precipitações pluviométricas, acima de 50 $\mathrm{mm}$ diários. Os indivíduos permaneciam nos corpos d'água efêmeros por poucos dias (no máximo quatro). Os machos apoiavam-se na base da vegetação (gramíneas principalmente), levantavam a região anterior do corpo e vocalizavam. Os indivíduos foram encontrados vocalizando em lagoas permanentes e áreas inundadas periodicamente (lagoa temporária e área de pastagem inundada), assim como em pequenas vazantes, onde o fluxo d'água era menor durante o dia. A desova constitui uma película fina na superfície de águas rasas e os ovos têm pólo animal preto e vegetativo cinza.

O diâmetro médio $( \pm \mathrm{s})$ dos óvulos maduros foi de $1,15 \pm 0,30 \mathrm{~mm}(\mathrm{n}=40)$ e o número médio de óvulos maduros por ovário foi de $620 \pm 251$ (n=39). As fêmeas foram, em média, maiores que os machos (tab. I), tanto em CRC quanto em massa corpórea. Os ovários maduros de E. bicolor pesaram em média 0,637 $\pm 0,24 \mathrm{~g}(\mathrm{n}=35)$, representando um investimento médio de $22,7 \pm 6,30 \%(\mathrm{n}=35)$ do peso do corpo em gônada. Foram encontradas relações positivas e significativas entre: CRC vs. NOM (fig. 1); PI vs. NOM (fig. 2); CRC vs. PO (fig. 3) e PI vs. PO (fig. 4).

Tabela I. Comparação da média do comprimento rostro-cloacal (CRC) (mm) e média da massa (g) de machos e fêmeas de Elachistocleis bicolor, coletados na Serra da Bodoquena, Mato Grosso do Sul, no período de outubro 2000 a setembro 2001.

\begin{tabular}{|c|c|c|c|c|c|c|c|c|}
\hline & \multicolumn{3}{|c|}{ Machos } & \multicolumn{3}{|c|}{ Fêmeas } & \multirow[b]{2}{*}{$\mathrm{t}$} & \multirow[b]{2}{*}{$\mathrm{p}$} \\
\hline & $\mathrm{n}$ & Méd. $\pm \mathrm{s}$ & Ampl. & $\mathrm{n}$ & Méd. $\pm \mathrm{s}$ & Ampl. & & \\
\hline CRC & 56 & $27,00 \pm 2,00$ & $22,9-31,5$ & 40 & $31,00 \pm 2,10$ & $27,2-35,5$ & 8,7 & $<0,001$ \\
\hline Massa & 56 & $1,82 \pm 0,48$ & $1,04-3,60$ & 35 & $2,15 \pm 0,56$ & $1,31-3,58$ & 3,0 & 0,003 \\
\hline
\end{tabular}



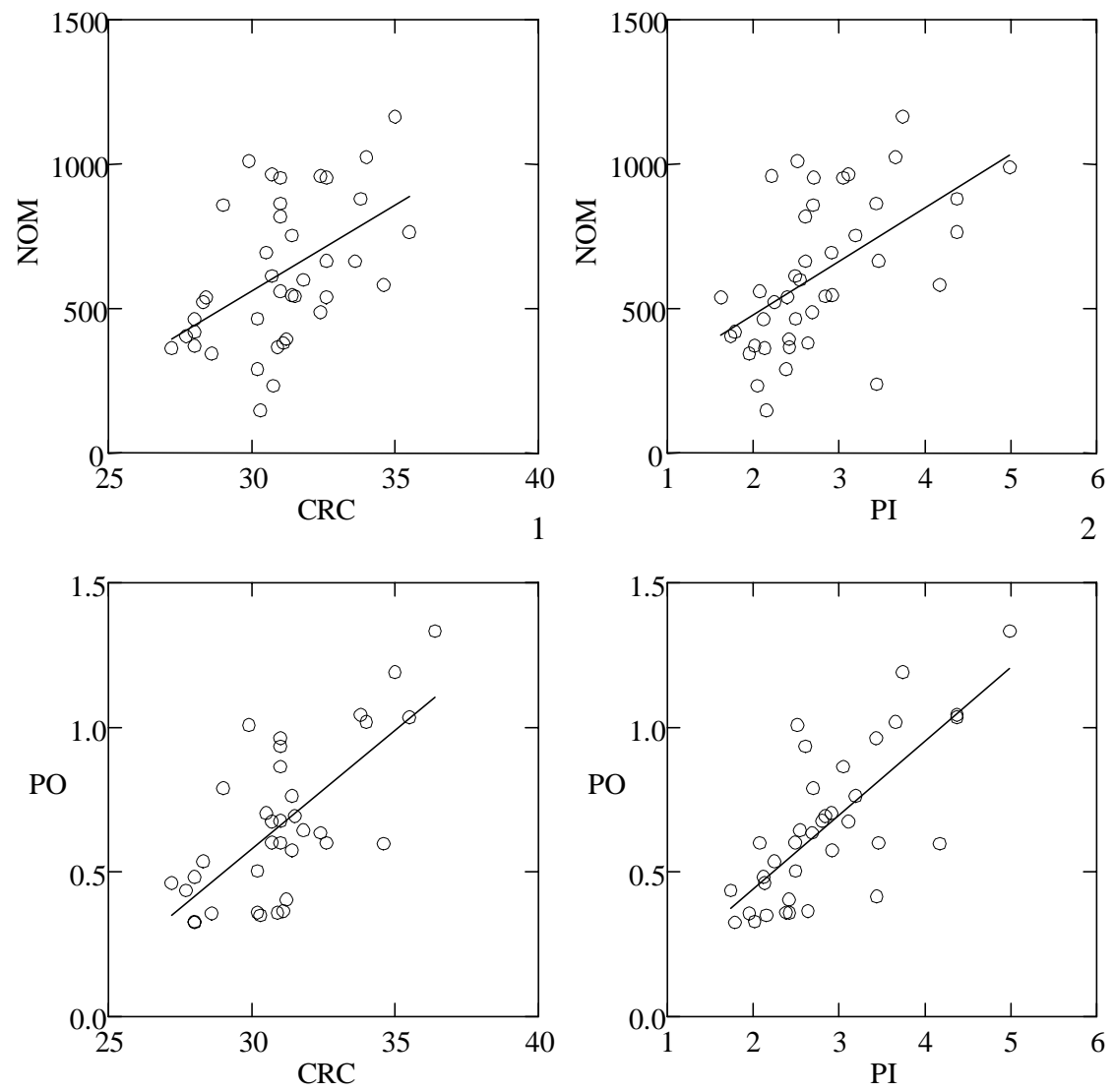

3

Figs. 1-4. Fêmeas de Elachistocleis bicolor, coletadas na Serra da Bodoquena, MS, período de outubro 2000 a setembro 2001, relações entre: 1, comprimento rostro-cloacal e número de óvulos maduros $\left(\mathrm{NOM}=59,591 \mathrm{CRC}-1227,2 ; \mathrm{n}=39 ; \mathrm{r}^{2}=0,25 ; \mathrm{p}=0,001\right) ; 2$, peso dos indivíduos e número de óvulos maduros $\left(\mathrm{NOM}=185,3 \mathrm{PI}+107,2 ; \mathrm{n}=41, \mathrm{r}^{2}=0,30 ; \mathrm{p}=0,002\right) ; 3$, comprimento rostro-cloacal e peso dos ovários $\left(\mathrm{PO}=0,082 \mathrm{CRC}-1,876 ; \mathrm{n}=35 ; \mathrm{r}^{2}=0,47 ; \mathrm{p}<0,01\right) ; 4$, peso dos indivíduos e peso dos ovários $\left(\mathrm{PO}=0,256 \mathrm{PI}-0,073 ; \mathrm{n}=36 ; \mathrm{r}^{2}=0,55 ; \mathrm{p}<0,01\right)$.

\section{DISCUSSÃO}

Apesar do período reprodutivo de E. bicolor estar vinculado à estação chuvosa (novembro a março), esta espécie não se reproduz continuamente, mas apenas quando há fortes chuvas (precipitação pluviométrica acima de $50 \mathrm{~mm}$ ) e em alguns dias subseqüentes (dois a três dias). Dentro da classificação proposta por WelLs (1977), E. bicolor é uma espécie próxima do padrão de reprodução explosiva. Este tipo de padrão reprodutivo já foi observado para outros microhilídeos, como Chiasmocleis 
ventrimaculata (Andersson, 1945), Ctenophryne geayi (Mocquard, 1904) (RodRíGuEZ \& Duellman, 1994) e para E. erythrogaster descrita por Kwet \& Di-BernARdo (1998).

Gallardo (1987) registrou a reprodução de E. bicolor na Argentina em setembro. KWET \& Di-BERNARDo (1999) relataram a reprodução de E. ovalis (Schneider, 1799) de outubro a fevereiro, na Serra Geral, no nordeste do Rio Grande do Sul. O comportamento e período reprodutivo observados por GALLARDo (1987) e KwET \& Di-BERNARDO (1999) foram semelhantes ao observado neste estudo.

As fêmeas de E. bicolor são, em média, maiores que os machos. Resultados semelhantes foram encontrados para E. ovalis e E. erythrogaster (KWET \& DIBernardo, 1999) e anuros de outras famílias (Martins, 1993; Bastos \& Haddad, 1996; Prado et al., 2000). Segundo Shine (1979), em 90\% das espécies de anuros conhecidas, as fêmeas são, em média, maiores que os machos e as causas desse fenômeno são diversas: fêmeas maiores são capazes de produzir óvulos maiores ou em maior quantidade (CRUMP \& KAPLAN 1979; PRADO et al., 2000); maior pressão de predação sobre machos maiores (HowARD, 1981), ou restrições ao crescimento de machos devido a demandas energéticas ligadas à atividade reprodutiva (WoOLBRIGHT, 1989).

As análises mostraram que $25 \%$ da variação do número de óvulos maduros e $47 \%$ do peso dos ovários foram explicados pelo CRC. Porém, $30 \%$ da variação do número de óvulos maduros e $55 \%$ do peso dos ovários foram explicados pelo peso dos indivíduos. Pradeiro \& Robinson (1990) e Prado et al. (2000) mostraram que o tamanho da fêmea está positivamente correlacionado com o número de ovos, ou óvulos, produzidos em algumas espécies de anuros. O maior tamanho do corpo confere vantagens reprodutivas às fêmeas, como aumento na produção ou no tamanho dos óvulos maduros (Crump, 1974; Howard, 1978, Prado et al., 2000). Fêmeas maiores têm maior chance de produzir mais de uma desova a cada período reprodutivo (Howard, 1978; Telford \& Dyson, 1990). Segundo Lang (1995), o volume é uma medida mais precisa para estimar o número de óvulos que o CRC. Howard (1988) registrou que entre os anuros o crescimento nunca cessa totalmente e que os indivíduos mais velhos são mais fecundos e apresentam maior sucesso reprodutivo. A análise da reta de regressão que se ajustou aos dados também demonstrou que o esforço reprodutivo das fêmeas menores foi proporcionalmente menor que o das maiores.

Agradecimentos. A C. P. A. Prado (Unesp, Rio Claro) e M. Tanaka (UFMS) pela revisão crítica do manuscrito; a T. S. F. de Souza, P. L. Filho, F. R. D. Fonseca e R. S. Arruda pela colaboração no trabalho de campo. À Coordenação do curso de Mestrado em Ecologia e Conservação (UFMS) pela utilização dos veículos. Ao CNPq pela bolsa de Mestrado ao autor sênior (processo 1311741/2000-4), e pelo auxílio financeiro ao projeto "Padrões de biodiversidade da fauna e flora do Pantanal" (processo 521746/97-3).

\section{REFERÊNCIAS BIBLIOGRÁFICAS}

Alvarenga, S. M.; Brasil, A. E. \& Del' Arco, D. M. 1982. Geomorfologia. In: IBGE. Folha SF21, Campo Grande. Rio de Janeiro. p. 125-184. (Levantamento de Recursos Naturais, 28). Bastos, R. P. \& Haddad, C. F. B. 1996. Breeding activity of the neotropical treefrog Hyla elegans (Anura, Hylidae). J. Herpetol., St. Louis, 30:355-360.

Beebee, T. J. C. 1996. Ecology and conservation of amphibians. London, Chapman \& Hall. 214p. 
Bertoluci, J. 1998. Annual patterns of breeding activity in Atlantic rainforest anurans. J. Herpetol., St. Louis, 32:607-611.

Boggiani, P. C. \& Clemente, J. 1999. A questão do licenciamento ambiental de empreendimentos turísticos no Planalto da Bodoquena, Mato Grosso do Sul. Revta Geogr., Dourados, (9):2432.

Colli, G. R.; Bastos, R. P. \& Araújo, A. F. B. 2002. The character and dynamics of the Cerrado herpetofauna. In: Oliveira, P. S. \& Marquis, R. J. ed. The Cerrados of Brazil. Ecology and natural history of a neotropical savanna. New York, Columbia University. p.223-241.

Crump, M. L. 1974. Reproductive strategies in a tropical anuran community. Misc. Publs Mus. Nat. Hist. Univ. Kans., Lawrence, 61:1-68.

Crump, M. L. \& Kaplan, R. H. 1979. Clutch energy partitioning of tropical tree frogs (Hylidae). Copeia, Lawrence, 1979:626-635.

De La Riva, I.; Köhler, J. et al. 2000. Ten years of research on Bolivian amphibians: updated checklist, distribution, taxonomic problems, literature and iconography. Revta Esp. Herp., Barcelona, 14:19-164.

De La Riva, I.; Márquez, R. \& Bosch, J. 1996. Advertisement calls of four microhylid frogs from Bolivia (Amphibia, Anura). Am. Midl. Nat., Indiana, 136(2):418-422.

Duellman, W. E. 1999. Patterns of distribution of amphibians in South America. In: A global perspective. London, John Hopkins University. p.255-328.

EmLen, S. T. 1968. A technique for marking anuran amphibians for behavioral studies. Herpetologica, Emporia, 24:172-173.

Freitas, E. F. L.; Spirandeli-Cruz, E. F. \& Jim, J. 2001. Comportamento reprodutivo de Leptodactylus fuscus (Schneider, 1799) (Anura: Leptodactylidae). Comun. Mus. Ciênc. Tecnol. PUCRS, Sér. Zool., Porto Alegre, 14(2):121-132.

Frost, D. R. 2002. Amphibian species of the world, version 2.20. The American Museum of Natural History. On line at http://research.amnh.org/herpetology/amphibia/index.html. 20.10.2002.

Furtado, P. P.; Guimarães J. G. \& Fonsar, B. C. 1982. Vegetação; estudo fitogeográfico. In: IBGE. Folha SF-21, Campo Grande. Rio de Janeiro. p.281-316. (Levantamento de Recursos Naturais, 28).

Gadgil, M. \& Bossert, W. H. 1970. Life historical consequences of natural selection. Am. Nat., Chicago, 104:1-24.

Gallardo, J. M. 1987. Anfibios argentinos: guía para su identificación. 1. ed. Buenos Aires, Agropecuaria. 98p.

HöDl, W. 1990. Reproductive diversity in Amazonian lowland frogs. In: Hanke, W. ed. Biology and physiology of amphibians. Stuttgart, Gustav Fisher Verlag. p.41-60.

How Evolution, Lancaster, 32:850-871.

1981. Sexual dimorphism in bullfrogs. Ecology, New York, 62(2):303-310.

1988. Reproductive success in two species of anurans. In: Clutton-Brock, T. H. ed. Reproductive success; studies of individual variation in contrasting breeding systems. Chicago, University of Chicago. p.99-113.

Juncá, F. A. 1998. Reproductive biology of Colostethus stepheni and Colostethus marchesianus (Dendrobatidae), with the description of a new anuran mating behavior. Herpetologica, Emporia, 54(3):377-387.

Kaplan, R. H. \& Salthe, S. N. 1979. The allometry of reproduction: an empirical view in salamanders. Am. Nat., Chicago, 113:671-689.

Kwet, A. \& Di-Bernardo, M. 1998. Elachistocleis erythrogaster, a new microhylid species from Rio Grande do Sul, Brazil. Stud. Neotrop. Fauna Environ., Lisse, 33:7-18.

1999. Pró-Mata Anfíbios. Porto Alegre, Edipucrs. 107p.

Lampo, M. \& Medialdea, V. 1996. Energy allocation patterns in Bufo marinus from two habitats in Venezuela. J. Trop. Ecol., Cambridge, 12:321-331.

LANG, C. 1995. Size-fecundity relationships among stream-breeding hylid frogs. Herpetol. Nat. Hist., Salt Lake City, 3(2):193-197.

Lima, P. A. \& Magnusson, W. E. 2000. Does foraging activity change with ontogeny? An 
assessment for six sympatric species of postmetamorphic litter anurans in Central Amazonia. J. Herpetol., St. Louis, 34(2):192-200.

Martins, M. 1993. Observations on the reproductive behavior of the smith frog, Hyla faber. Herpetol. J., London, 3:31-34.

Pombal, J. P., JR. \& Bastos, R. P. 1996. Uma nova espécie do gênero Scinax Wagler, 1830, do Brasil central (Amphibia, Anura, Hylidae). Bolm Mus. nac. Rio de J., Rio de Janeiro, 371:1-11.

Pough, F. H.; Andrews, R. M. et al. 1998. Herpetology. New Jersey, Prentice-Hall. 577p.

Pradeiro, M. J. \& Robinson, M. D. 1990. Reproduction in the toad Colostethus trinitatis (Anura: Dendrobatidae) in a northern Venezuela seasonal environment. J. Trop. Ecol., Cambridge, 6:333-341.

Prado, C. P. A.; Uetanabaro, M. \& Lopes, F. S. 2000. Reproductive strategies of Leptodactylus chaquensis and Leptodactylus podicipinus in the Pantanal, Brazil. J. Herpetol., St. Louis, 34(1): $135-139$.

Rodríguez, L. O. \& Duellman, W. E. 1994. Guide to the frogs of the Iquitos Region, Amazonian Peru. Lawrence, University of Kansas. 80p.

Shine, R. 1979. Sexual selection and sexual dimorphism in the Amphibia. Copeia, Lawrence, 1979:297-306.

Stearns, S. C. 1992. The evolution of life histories. New York, Oxford University. 249p.

Strüssmann, C.; Prado, C. P. A. et al. 2000. Amphibians recorded during the AquaRAP survey of localities in the southern Pantanal floodplains and surrounding Cerrado, MS, Brasil. In: Willink, P.; Chernoff, B. et al. Rapid assessment program, bulletin of biological assessment. A biological assessment of the aquatic ecosystems of the Pantanal, Campo Grande, Mato Grosso do Sul, Brasil. Washington, Conservation International. $305 \mathrm{p}$.

Telford, S. R. \& Dyson, M. L. 1990. The effect of rainfall on interclutch interval in painted reed frogs (Hyperolius marmoratus). Copeia, Lawrence, 1990(3):644-648.

Wells, K. D. 1977. The social behavior of anuran amphibians. Anim. Behav., London, 25:666-693.

Woolbright, L. L. 1989. Sexual dimorphism in Eleutherodactylus coqui: selection pressures and growth rates. Herpetologica, Emporia, 45:68-74.

ZAR, J. H. 1984. Biostatistical analysis. 2. ed. New Jersey, Prentice Hall. 718p.

Recebido em 14.08.2002; aceito em 26.06.2003. 